\title{
THE ADDITION THEOREM FOR THE ENTROPY OF TRANSFORMATIONS OF $G$-SPACES( $\left.{ }^{1}\right)$
}

\author{
BY \\ R. K. THOMAS
}

\begin{abstract}
For a measure-preserving transformation $T$ which is a skew-product of a measure-preserving transformation $S$ and a topological group endomorphism $\sigma$, it is shown that the entropy $h$ satisfies the following "addition theorem": $h(T)=h(S)+h(\sigma)$.
\end{abstract}

Introduction. In a previous paper [4], conditions were given for a certain type of transformation of a $G$-space ( $G$ being a compact separable group) to have completely positive entropy. It is useful to be able to calculate the actual numerical value of the entropy; the purpose of the present paper is to extend previously known formulae to cover this type of transformation.

As in [4], the notation of Rohlin's survey article [3] is used: the entropy of a measure-preserving transformation $T$ of a Lebesgue space $(M, \mathscr{B}, \mu)$ is denoted by $h(T) ; H(\xi)$ denotes the entropy of the (measurable) partition $\xi$ of $M$ and $H(\xi / \eta)$ denotes the mean conditional entropy of $\xi$ with respect to $\eta$.

Throughout this paper, the basic measure space $(M, \mathscr{B}, \mu)$ will be a direct product of a Lebesgue space $(X, \mathscr{C}, \nu)$ and a compact separable group $G$ with Borel sets and Haar measure $m$ (this also being a Lebesgue space); all the measures are normalized, i.e. $\mu(M)=\nu(X)=m(G)=1$. The measure-preserving transformation $T$ will act as follows:

$$
T(x, g)=(S x, \sigma(g) \varphi(x)),
$$

where $S$ is a measure-preserving transformation of $X, \sigma$ is a group endomorphism of $G$ and $\varphi: X \rightarrow G$ is some measurable map; throughout this paper, such a transformation will be described as a skew-product of $S$ and $\sigma$ (the map $\varphi$ not being specified).

It will be proved that

$$
h(T)=h(S)+h(\sigma) .
$$

For the case where $M$ is itself a compact separable group, $T$ is a group endomorphism and $G$ is a $T$-invariant $(T G \subset G)$ closed normal subgroup ( $\sigma=$ the restriction of $T$ to $G$ ), this result was proved by Juzvinskir in [2] as an essential step

Received by the editors March 18, 1970 and, in revised form, October 27, 1970.

AMS 1970 subject classifications. Primary 28A65, 22D40; Secondary 22D05, $22 \mathrm{E} 15$.

Key words and phrases. Skew-product transformation, addition theorem, rigid groups.

(1) This paper is part of a thesis submitted at the University of Warwick for a Ph.D. degree.

Copyright (C) 1971, American Mathematical Society 
in his proof that an ergodic endomorphism of a compact separable group has completely positive entropy-the result generalized by the present author in [4]; (1) was not needed there but it is a very useful result in its own right.

The present paper leans heavily on Juzvinskir's work which is adapted, where possible, to fit the broader context; the "proof by steps" technique (also used by Rohlin in [6]) and a number of theorems are taken from it-acknowledgement is given in each instance. The essential step in the generalization is $\$ 2.5$ (a compact Lie group is rigid). This also leads to a simplification in the proof as does a new treatment of Bernoulli endomorphisms (§3). Following Juzvinskiř, (1) will be called "The Addition Theorem".

Note that more restrictive conditions are given here for $T$ than were given in [4] (skew-product as opposed to $\sigma$-commuting with $G$-action); this is necessary for the elimination of trivial group action (if this were allowed, $\sigma$ and consequently $h(\sigma)$ could be practically anything for a given system) and does not seriously limit applications.

My thanks are due to Dr. W. Parry for supervising this work.

\section{Preliminaries.}

1.1. Standard results from entropy theory. (i) If $\xi_{1} \leqq \xi_{2} \leqq \cdots$ is a sequence of $T$-invariant $\left(T^{-1} \xi_{n} \leqq \xi_{n}\right)$ partitions of $M$ such that $\bigvee_{n} \xi_{n}=\varepsilon$, then $h\left(T_{\xi_{1}}\right) \leqq h\left(T_{\xi_{2}}\right)$ $\leqq \cdots$ and $\lim _{n \rightarrow \infty} h\left(T_{\xi_{n}}\right)=h(T)$, where $T_{\xi_{n}}$ is the factor-transformation induced by $T$ in the Lebesgue space $M / \xi_{n}$ consisting of elements of $\xi_{n}[3, \S 9.6]$. ( $\varepsilon$ always denotes the partition of the space being considered into distinct points.)

(ii) For any two partitions $\xi$ and $\eta$ with finite entropy (in particular, if the partitions are finite),

$$
|H(\xi)-H(\eta)| \leqq H(\xi / \eta)+H(\eta / \xi) \quad[3, \S 6.5] .
$$

(iii) For any three partitions $\xi, \eta$ and $\zeta, h(\xi \vee \eta / \zeta) \leqq H(\xi / \zeta)+H(\eta / \zeta)[3, \S 5.6]$,

$$
H(\xi / \eta \vee \zeta) \leqq H(\xi / \zeta) \quad[3, \S 5.10]
$$

(iv) $h\left(T^{n}\right)=n h(T)[3, \S 9.3]$.

(v) In [1], Abramov and Rohlin proved that the entropy of any skew-product transformation is given by

$$
h(T)=h(S)+h_{S}(\tau),
$$

where $T$ and $S$ are as above, $\tau=\left\{\tau_{x}\right\}$ is a collection of measure-preserving transformations of the second factor-for the purposes of this paper,

$$
\tau_{x}(g)=\sigma(g) \varphi(x)
$$

and $h_{S}(\tau)$ is a quantity known as the "mixed entropy" of $\tau$.

If $\xi_{1} \leqq \xi_{2} \leqq \cdots$ is an increasing sequence of finite partitions of the second factor 
( $G$ in this case) such that $\bigvee_{n} \xi_{n}=\varepsilon$, then

$$
h_{S}(\tau)=\lim _{m \rightarrow \infty} \lim _{k \rightarrow \infty} \frac{1}{k} \int_{X} H\left(\xi_{x, m}^{k}\right) d \nu
$$

where $\xi_{x, m}^{k}=\bigvee_{i=0}^{k-1} \tau_{x}^{-1} \cdot \tau_{S x}^{-1} \cdot \cdots \cdot \tau_{S^{i}-1}^{-1} \xi_{x}$

(3) should be compared with the following formula for $h(\sigma)$ to be used later:

$$
h(\sigma)=\lim _{m \rightarrow \infty} \lim _{k \rightarrow \infty} \frac{1}{k} H\left(\xi_{m}^{k}\right),
$$

where $\xi_{m}^{k}=\bigvee_{\substack{k=0 \\ i=0}}^{-i} \sigma^{-i} \xi_{m}$ and the sequence $\left\{\xi_{n}\right\}$ is as before. (4) follows from [3, $\S 9.5$ and §7.3].

Clearly, formula (3) will be very important in the proof of the addition theoremall that remains is to prove that $h_{S}(\tau)=h(\sigma)$. Alas, this is not as easy as it might at first appear. In the case where $T$ is a direct product (i.e. $\varphi(x)=e$ for all $x$ in $X$ ), $h_{S}(\sigma)=h(\sigma)$ by $[1, \S 2.4]$.

1.2. Subgroups of $G$. Let $H$ be a completely $\sigma$-invariant $(\sigma H=H)$ closed subgroup of $G$. $\zeta(H)$ denotes the partition of $M$ each element of which is the direct product of a point from $X$ with a right coset of $H ; \sigma_{H}$ denotes the restriction of $\sigma$ to $H ; \sigma_{G / H}$ denotes the transformation induced by $\sigma$ on the right coset space $G / H$ and $T_{\zeta(H)}$ denotes the factor-transformation induced by $T$ on $M / \zeta(H), \zeta(H)$ being invariant under $T$.

There exists a Borel cross-section $\psi$ which takes almost every coset $\mathrm{Hg}$ to a single point $\psi(g) \in H g ; \psi(G)$ is a Borel subset $Y \subset G$. Measure theoretically, $G$ is the direct product of $Y$ and $H$ ( $Y$ having the measure induced by $m$ and $H$ having its own normalized Haar measure). $\psi \cdot \sigma$ is isomorphic to and will be identified with $\sigma_{G / H}$. Thus $\sigma$ becomes the skew-product of $\sigma_{G / H}$ and $\sigma_{H}$. Similarly, as $M$ is the direct product of $(X \otimes Y)$ and $H, T$ can also be regarded as being a skew-product of $T_{\zeta(H)}$ and $\sigma_{H}$.

In order to deal with situations in which $\sigma$ is not onto (when a subgroup is properly mapped into itself), Juzvinskir in [2] defined $h(\sigma)$ by putting $h(\sigma)=h\left(\sigma_{G^{\prime}}\right)$, where $G^{\prime}=\bigcap_{n} \sigma^{n} G$. It turns out that $h(S)=h\left(T_{\zeta\left(G^{\prime}\right)}\right)$ and so one could say that $h(T)=h(S)+h(\sigma)$ if one knew that $h(T)=h\left(T_{\zeta\left(G^{\prime}\right)}\right)+h\left(\sigma_{G^{\prime}}\right)$. All this goes over to generalization but it is felt that its value is limited-it does not make much sense to consider the entropy of a non-measure-preserving transformation. As it is sufficient to consider only the entropy of endomorphisms restricted to completely invariant subgroups, only skew-products of a measure-preserving transformation and a group endomorphism mapping a group onto itself will be considered.

1.3. Proof by steps. Let $H$ be a closed completely-invariant normal subgroup of $G$. Clearly, $T_{\zeta(H)}$ is a skew-product of $S$ and $\sigma_{G / H}$.

Suppose that the addition theorem has been proved for endomorphisms of the 
types of $\sigma_{G / H}$ and $\sigma_{H}$ or, as will be stated in the future, that one has the addition theorem for $\sigma_{G / H}$ and for $\sigma_{H}$ (no limitations on $S$ ), then

and

$$
\begin{aligned}
h\left(T_{\zeta(H)}\right) & =h(S)+h\left(\sigma_{G / H}\right), \\
h(T) & =h\left(T_{\zeta(H)}\right)+h\left(\sigma_{H}\right)
\end{aligned}
$$

$$
h(\sigma)=h\left(\sigma_{G / H}\right)+h\left(\sigma_{H}\right) .
$$

Hence,

$$
h(T)=h(S)+h\left(\sigma_{G / H}\right)+h\left(\sigma_{H}\right)=h(S)+h(\sigma) .
$$

So, the addition theorem for $\sigma$ can be proved by finding a suitable completely $\sigma$-invariant closed normal subgroup $H$ and then proving it for the two "steps" $\sigma_{G / H}$ and $\sigma_{H}$.

This principle can clearly be extended to a finite number of steps: suppose that $G$ contains a sequence $G=G_{0} \supset G_{1} \supset G_{2} \supset \ldots \supset G_{n}=e$ of completely $\sigma$-invariant closed subgroups with $G_{i+1}$ normal in $G_{i}$; the addition theorem for $\sigma$ can be proved by proving it for all the steps $\sigma_{G_{i} / G_{i+1}}$. The next subsection shows that the number of steps can be infinite.

1.4. Taking limits. Suppose that $G$ contains a sequence $G=G_{0} \supset G_{1} \supset G_{2} \supset \ldots$ of $\sigma$-invariant $\left(\sigma G_{n} \subset G_{n}\right)$ closed subgroups such that $\bigcap_{n} G_{n}=e$ and that

$$
h\left(T_{\zeta\left(G_{n}\right)}\right)=h(S)+h\left(\sigma_{G / G_{n}}\right) \text { for all } n \text {. }
$$

By $§ 1.1,(\mathrm{i})$,

$$
h(T)=\lim _{n \rightarrow \infty} h\left(T_{\zeta\left(G_{n}\right)}\right) \text { and } h(\sigma)=\lim _{n \rightarrow \infty} h\left(\sigma_{G / G_{n}}\right)
$$

Hence,

$$
h(T)=h(S)+h(\sigma) .
$$

So (5) implies the addition theorem for $\sigma$.

\section{Rigid groups.}

2.1. Notation. Suppose that $\xi=\left\{A_{\alpha}\right\}$ is a partition of a measurable set $B \subset G$.

For any $g$ in $G, \xi g$ will denote the partition of $B g$ into $\left\{A_{\alpha} g\right\}$.

For a measurable set $C \subset G, \xi-C$ will denote the partition of $B-C$ into $\left\{\left(A_{\alpha}-C\right)\right\}$.

2.2. Definition. The group $G$ is said to be rigid if there exist an increasing sequence $\xi_{1} \leqq \xi_{2} \leqq \cdots$ of finite partitions of $G$ and a real number $Q$ such that $\bigvee_{n} \xi_{n}=\varepsilon$ and $H\left(\xi_{n} g / \xi_{n}\right) \leqq Q$ for all $n$.

2.3. THEOREM. The addition theorem holds for an endomorphism $\sigma$ of a rigid group $G$.

Proof (adapted from Juzvinskir $[2, \S 7.4])$. By $§ 1.1,(v)$, it is sufficient to prove that $h_{\mathrm{S}}(\tau)=h(\sigma)$. As

$$
T(x, g)=(S x, \sigma(g) \varphi(x))=\left(S x, \tau_{x}(g)\right) \quad\left(\tau=\left\{\tau_{x}\right\}\right),
$$


$T^{n}$ can be expressed in the form:

$$
T^{n}(x, g)=\left(S^{n} x, \sigma^{n}(g) \varphi_{n}(x)\right)=\left(S^{n} x, \tau_{n, x}(g)\right) \quad\left(\tau_{n}=\left\{\tau_{n, x}\right\}\right) .
$$

Now $h\left(T^{n}\right)=h\left(S^{n}\right)+h_{S^{n}}\left(\tau_{n}\right)$ (applying the formula for the entropy of a skewproduct, $\S 1.1,(\mathrm{v}))$ and so $n h(T)=n h(S)+h_{S^{n}}\left(\tau_{n}\right)$ (applying $\S 1.1$, (iv)). Hence,

$$
h_{\mathrm{S}^{n}}\left(\tau_{n}\right)=n h_{\mathrm{S}}(\tau)
$$

(comparing the previous formula with (2)). $\tau_{n, x}(g)=\sigma^{n}(g) \varphi_{n}(x)$ and so $\tau_{n, x}^{-1} \eta$ $=\sigma^{-n}\left(\eta \cdot\left[\varphi_{n}(x)\right]^{-1}\right)$ for any partition $\eta$ of $G$. Applying formulae (3) and (4) of 1.1, (v),

$$
\begin{aligned}
\left|h_{S^{n}}\left(\tau_{n}\right)-h\left(\sigma^{n}\right)\right| & =\lim _{m \rightarrow \infty} \lim _{k \rightarrow \infty} \frac{1}{k}\left|\int_{X}\left(H\left(\xi_{x, m}^{k, n}\right)-H\left(\xi_{m}^{k, n}\right)\right) d \nu\right| \\
& \leqq \lim _{m \rightarrow \infty} \lim _{k \rightarrow \infty} \frac{1}{k} \int_{X} \Delta_{x, m}^{k, n} d \nu,
\end{aligned}
$$

where

$$
\begin{aligned}
\Delta_{x, m}^{k, n} & =H\left(\xi_{x, m}^{k, n}\right)-H\left(\xi_{m}^{k, n}\right) \\
\xi_{x, m}^{k, n} & =\bigvee_{i=0}^{k-1} \tau_{n, x}^{-1} \cdot \tau_{n, S}^{-1} \cdot \cdots \cdot \tau_{n, S^{-1}}^{-1} \xi_{m} \quad\left(\left\{\xi_{m}\right\}\right. \text { as in Definition 2.2) } \\
& =\bigvee_{i=0}^{k-1} \sigma^{-n i}\left(\xi_{m} \cdot\left[\varphi_{n}\left(S^{i-1} x\right)\right]^{-1} \cdots \cdot\left[\varphi_{n}(x)\right]^{-1}\right) \\
& =\bigvee_{i=0}^{k-1} \sigma^{-n i}\left(\xi_{m} \cdot f_{n, i}(x)\right) \quad(\text { simplifying notation), } \\
\xi_{m}^{k, n} & =\bigvee_{i=0}^{k-1} \sigma^{-n i} \xi_{m} .
\end{aligned}
$$

Using 1.1, (ii),

$$
\Delta_{x, m}^{k, n} \leqq H\left(\bigvee_{i=0}^{k-1} \sigma^{-n i}\left(\xi_{m} \cdot f_{n, i}(x)\right) / \bigvee_{i=0}^{k-1} \sigma^{-n i} \xi_{m}\right)+H\left(\bigvee_{i=0}^{k-1} \sigma^{-n i} \xi_{m} / \bigvee_{i=0}^{k-1} \sigma^{-n i}\left(\xi_{m} \cdot f_{n, i}(x)\right)\right) \text {, }
$$

which can be expanded by 1.1 , (iii), to give

$$
\begin{aligned}
\Delta_{x, m}^{k, n} & \leqq \sum_{i=0}^{k-1}\left[H\left(\sigma^{-n i}\left(\xi_{m} \cdot f_{n, i}(x)\right) / \sigma^{-n i} \xi_{m}\right)+H\left(\sigma^{-n i} \xi_{m} / \sigma^{-n i}\left(\xi_{m} \cdot f_{n, i}(x)\right)\right)\right] \\
& =\sum_{i=0}^{k-1}\left[H\left(\xi_{m} \cdot f_{n, i}(x) / \xi_{m}\right)+H\left(\xi_{m} \cdot\left[f_{n, i}(x)\right]^{-1} / \xi_{m}\right)\right] \\
& \leqq 2 k Q \quad \text { by the definition of a rigid group. }
\end{aligned}
$$

Hence, it follows that

$$
\left|h_{S^{n}}\left(\tau_{n}\right)-h\left(\sigma^{n}\right)\right| \leqq 2 Q .
$$

This combined with (6) and $\$ 1.1$, (iv), gives

$$
\left|h_{S}(\tau)-h(\sigma)\right| \leqq 2 Q / n \text { for all } n .
$$

So $h_{s}(\tau)$ must equal $h(\sigma)$. 
2.4. Totally disconnected groups. A search for rigid groups is begun now. It is easy to prove that a compact separable totally disconnected group $G$ is rigid:

Proof (Juzvinskii $[2, \S 7.2]) . G$, being totally disconnected, contains a sequence $G=G_{0} \supset G_{1} \supset G_{2} \supset \ldots$ of open normal subgroups such that $\bigcap_{n} G_{n}=e$. Let $\xi_{n}$ be the partition of $G$ into cosets of $G_{n}$; then $\xi_{n} g=\xi_{n}$ for all $g$ in $G$ and all $n$. Therefore, $H\left(\xi_{n} g / \xi_{n}\right)=0$ for all $g$ and all $n$ and so $\xi_{1} \leqq \xi_{2} \leqq \cdots$ satisfies all the requirements of Definition 2.2 with $Q=0$.

2.5. Lie groups. A compact Lie group also turns out to be rigid but this is more difficult to prove. It is, however, a crucial result. First some simple observations.

Note. The maximum number of disjoint $p$-dimensional (open) balls of radius $r$ that can intersect a single $p$-ball of radius $R$ depends only on $p$ and the ratio $r / R=s$. This number will be denoted by $I(p, s)$.

Definition. A sequence $\xi_{1} \leqq \xi_{2} \leqq \cdots$ of finite partitions of the group $G$ such that $\bigvee_{n} \xi_{n}=\varepsilon$ will be said to satisfy the "bounded intersection condition" if the number of elements of $\xi_{n} g$ which intersect a single element of $\xi_{n}$ (in sets of positive measure) is bounded above by some number $N$ for all $g$ in $G$ and all $n$.

LEMma. The existence of a sequence of partitions $\left\{\xi_{n}\right\}$ satisfying the bounded intersection condition implies that $G$ is rigid.

Proof. For a finite partition $\xi=\left\{A_{i}\right\}$ of $G$ and $g$ in $G$, the definition of conditional entropy gives

$$
H(\xi g / \xi)=\sum_{i} m\left(A_{i}\right) \cdot H\left(\xi g, A_{i}\right)
$$

where

$$
H\left(\xi g, A_{i}\right)=-\sum_{j} \frac{m\left(A_{j} g \cap A_{i}\right)}{m\left(A_{i}\right)} \log \frac{m\left(A_{j} g \cap A_{i}\right)}{m\left(A_{i}\right)}
$$

is the entropy of the partition of $A_{i}$ into the collection of sets $\left\{A_{j} g \cap A_{i}\right\}_{j}$.

$H\left(\xi g, A_{i}\right) \leqq \log m_{i}$, where $m_{i}$ is the number of elements of the partition $\left\{A_{j} g \cap A_{i}\right\}_{j}$ (sets of measure zero not being counted) [3, \$4.7]. It follows that $H(\xi g / \xi) \leqq \log m$, where $m=\max _{i} m_{i}$.

Hence for the sequence $\left\{\xi_{n}\right\}$ satisfying the conditions of the definition, $H\left(\xi_{n} g / \xi_{n}\right)$ $\leqq \log N$ for all $n$ and all $g$ in $G$ and so the conditions of Definition 2.2 are satisfied with $Q=\log N$.

THEOREM. Any compact Lie group $G$ admits a sequence $\xi_{1} \leqq \xi_{2} \leqq \cdots$ of finite partitions such that $\bigvee_{n} \xi_{n}=\varepsilon$ and the bounded intersection condition is satisfied.

Proof. First it is assumed that $G$ is connected. $G$ has a Riemannian structure which is invariant under both left and right translation [5, p. 188] and the resulting metric $d$ has the following property ([5, Chapter I, Propositions 9.9 and 9.10]): 
There exists an $\varepsilon>0$ such that, for $A$ and $B$ in the tangent plane $T G_{e}$ at the identity and $\|A\|<\varepsilon$ and $\|B\|<\varepsilon, N_{\varepsilon}$ is a normal neighborhood of $e$ and

$$
\|A-B\| / d(a, b) \rightarrow 1 \quad \text { as }(a, b) \rightarrow(e, e),
$$

where $a=\operatorname{Exp}_{e} A, b=\operatorname{Exp}_{e} B$ and $N_{\varepsilon}$ is the spherical neighborhood of $e$ of radius $\varepsilon$ (w.r.t. $d$ ).

$\delta$ is chosen now sufficiently small (smaller than $\varepsilon / 4 \sqrt{ } p$, where $p$ is the dimension of $G)$ for $\frac{1}{2}<(A-B) / d(a, b)<2$ for $a$ and $b$ in $N_{4 \delta \downarrow}$.

A (open) cube $C$ of edge $\delta$ with one corner at $0\left(\operatorname{Exp}_{e} 0=e\right)$ is constructed in $T G_{e}$. $C_{n}$ will denote the partition of $C$ into $(n !)^{p}$ equal (open) cubes $C_{n 1}, C_{n 2}, \ldots$ each of edge $\delta / n$ ! (a set of measure zero has been discarded). Let $D=\operatorname{Exp}_{e} C$ and $D_{n}$ $=\operatorname{Exp}_{e} C_{n}$ (i.e. $D_{n}$ is the partition of $D$ into $\left\{\operatorname{Exp}_{e} C_{n i}\right\}$ ).

As $G$ is compact, a finite number of translations of $D\left(D, D a_{1}, D a_{2}, \ldots, D a_{m-1}\right.$ say) cover $G$. The partition $\xi_{n}$ is formed by taking the elements of $D_{n}, D_{n} a_{1}-D$, $D_{n} a_{2}-\left(D \cup D a_{1}\right), \ldots, D_{n} a_{m-1}-\left(D \cup D a_{1} \cup \ldots \cup D a_{m-2}\right)$; in other words, $m$ copies of $D_{n}$ are fitted together so that there is no overlapping (notation as in \$2.1). Clearly, $\left\{\xi_{n}\right\}$ is an increasing sequence of finite partitions satisfying $\bigvee_{n} \xi_{n}=\varepsilon$; it remains to show that $\left\{\xi_{n}\right\}$ satisfies the bounded intersection condition.

The intersection of $D_{n} g$ with $D_{n}$ for any $g$ in $G$ is considered: if this intersection is nonempty, $D_{n} g \subset N_{4 \delta \sqrt{ } p}$ and then $E_{n}(g)=\operatorname{Exp}_{e}^{-1} D_{n} g$ can be considered:

Each element of $C_{n}$ contains a ball of diameter $\delta / n$ ! and is contained in a ball of diameter $\delta \sqrt{ } p / n$ !. Hence, each element of $D_{n}$ contains a ball of diameter $\delta / n ! 2$ and is contained in a ball of diameter $2 \delta \sqrt{ } p / n$ !. As $d$ is invariant under translation, the elements of $D_{n} g$ have the same property and so if $E_{n}(g)$ is defined, then each element of $E_{n}(g)$ contains a ball of diameter $\delta / n ! 4$ and is contained in a ball of $4 \delta \sqrt{ } p / n !$.

It follows that not more than

$$
I\left(p, \frac{\delta}{n ! 4} / \frac{\delta \sqrt{ } p}{n !}\right)=I(p, 1 / 4 \sqrt{ } p)
$$

elements of $E_{n}(g)$ can intersect a single element of $C_{n}$ and hence that not more than $I(p, 1 / 4 \sqrt{ } p)$ elements of $D_{n} g$ can intersect a single element of $D_{n}$.

As $\xi_{n}$ is constructed from $m$ copies of $D_{n}$, not more than $m I(p, 1 / 4 \sqrt{ } p)$ elements of $\xi_{n} g$ can intersect a single element of $\xi_{n}$ for all $g$ in $G$ and all $n$. Thus $\left\{\xi_{n}\right\}$ satisfies the bounded intersection condition.

The extension of the proof to a finite number of connected components is trivial.

2.6. Abelian groups of finite dimension. Finite-dimensional tori were covered by the last section; the following theorem also includes a wider class of abelian groups.

TheOREM (JuzVInSKIǏ $[2, \S 7.3]$ ). A compact connected abelian group $G$ of finite dimension $p$ is rigid. 
Proof (adapted from [2]). $G$ contains a sequence $G=G_{0} \supset G_{1} \supset G_{2} \supset \ldots$ of closed subgroups such that $\bigcap_{m} G_{m}=e$ and $G / G_{m}$ is isomorphic to some $p$-dimensional torus $A$ for all $m$. Let $P_{m}: G \rightarrow A$ be the map obtained by composing the projection of $G$ onto $G / G_{m}$ with the isomorphism of $G / G_{m}$ onto $A$.

Using the notation of the proof of the last theorem, $\operatorname{Exp}_{e}$ is an isometry for the torus $A$ and $D$ is taken to be the whole of $A$ (represented as a $p$-cube). So the partitions $\left\{P_{m}^{-1} D_{n}\right\}$ are finite and satisfy

$$
H\left(\left(P_{m}^{-1} D_{n}\right) g / P_{m}^{-1} D_{n}\right)=H\left(D_{n} \cdot P_{m} g / D_{n}\right) \leqq \log I(p, 1 / 4 \sqrt{ } p)
$$

(see the proof of lemma in $\$ 2.5$; “ $N "=I(p, 1 / 4 \sqrt{ } p)$ from the proof of the last theorem).

An increasing sequence of integers $n(1)=1, n(2)=2, n(3), n(4), \ldots$ is constructed inductively so that $\left\{\xi_{m}=P^{-1} D_{n(m)}\right\}$ is an increasing sequence; it is assumed that all the $n(r)$ 's have been chosen for $r \leqq k . P_{k} P_{k+1}^{-1}$ is an endomorphism of $A$ onto $A$ and so has a kernel of finite order $s$; the inverse map $P_{k+1} P_{k}^{-1}$ takes a single cube from $D_{n(k)}$ to the union of $s$ disjoint identical cuboids. It is easy to see that $D_{s n(k)}$ $\geqq P_{k+1} P_{k}^{-1} D_{n(k)}$ from which it follows that $P_{k+1}^{-1} D_{s n(k)} \geqq P_{k}^{-1} D_{n(k)}$. So $n(k+1)$ is put equal to $s n(k)$.

The sequence $\left\{\xi_{m}\right\}$ so constructed satisfies all the conditions of Definition 2.2 and so $G$ is rigid.

3. Bernoulli group automorphisms and endomorphisms. In the last section, the addition theorem was proved for endomorphisms of totally disconnected groups, Lie groups and finite-dimensional abelian groups. In order to be able to use the theory of the structure of compact groups to tie these results together to give the addition theorem for an endomorphism of an arbitrary compact group $G$, it is necessary to prove the addition theorem for one more class of endomorphisms, namely Bernoulli group automorphisms and endomorphisms.

3.1. Definitions. Let $G$ be a direct product of a two-way (one-way) infinite sequence of copies of some compact group $G_{0}$ known as the group of states; an element $g$ can be represented as a sequence $\left\{g_{i}\right\}_{-\infty}^{\infty}\left(\left\{g_{i}\right\}_{0}^{\infty}\right)$. A Bernoulli group automorphism (endomorphism) takes the sequence $\left\{g_{i}\right\}_{-\infty}^{\infty}\left(\left\{g_{i}\right\}_{0}^{\infty}\right)$ to the sequence $\left\{h_{i}\right\}_{-\infty}^{\infty}\left(\left\{h_{i}\right\}_{0}^{\infty}\right)$, where $h_{i}=g_{i+1}$.

In some cases $G$ will be rigid (if $G_{0}$ is finite for example) but the following theorem covers all possibilities.

3.2. THEOREM. When $\sigma$ is a Bernoulli group endomorphism of the group $G, T$ is isomorphic to the direct product of $S$ and $\sigma$.

Proof. $T(x, g)=(S x, \sigma(g) \varphi(x))$; in this case, $\varphi(x)$ is a sequence $\left\{\varphi_{i}(x)\right\}_{0}^{\infty}$. Let $F: M \rightarrow M$ be the invertible measure-preserving transformation given by $F(x, g)$ $=(x, g \cdot f(x))$, where $f=\left\{f_{i}\right\}_{0}^{\infty}$ is some measurable function from $X$ to $G$.

$$
F^{-1} T F(x, g)=\left(S x, \sigma(g) \sigma(f(x)) \varphi(x)[f(S x)]^{-1}\right)
$$


and so if

$$
\sigma(f(x)) \varphi(x)[f(S x)]^{-1}=e
$$

for all $x$ in $X$, then $S \otimes \sigma=F^{-1} T F$, i.e. $T$ is isomorphic to $S \otimes \sigma$.

(7) is equivalent to the set of equations

$$
f_{i+1}(x) \varphi_{i}(x)=f_{i}(S x) .
$$

The equations (8) are satisfied by

$$
\begin{aligned}
f_{0}(x) & =e \\
f_{1}(x) & =\left[\varphi_{0}(x)\right]^{-1} \\
f_{2}(x) & =\left[\varphi_{0}(S x) \varphi_{1}(x)\right]^{-1} \\
f_{i}(x) & =\left[\varphi_{0}\left(S^{i-1} x\right) \varphi_{1}\left(S^{i-2} x\right) \cdots \varphi_{i-1}(x)\right]^{-1} .
\end{aligned}
$$

As the $f_{i}$ 's are all products of measurable functions, the measurability requirements for $f$ and $F$ are satisfied and so the map $F$, given by these solutions of (8), gives an isomorphism between $T$ and $S \otimes \sigma$.

COROllaRY. For $\sigma$ a Bernoulli group automorphism or endomorphism of the group $G, h(T)=h(S)+h(\sigma)$.

Proof. When $\sigma$ is a Bernoulli group endomorphism, $T$ is isomorphic to a direct product and so has the same entropy $[3, \S 16.3]$ :

$$
h(T)=h(S \otimes \sigma)=h(S)+h(\sigma) \quad(\S 1.1,(\mathrm{v})) .
$$

For a Bernoulli group automorphism, let $G_{n}$ be the closed normal subgroup of $G$ consisting of all sequences $\left\{g_{i}\right\}_{-\infty}^{\infty}$ for which $g_{k}=e$ for $k \geqq-n, n=0,1, \ldots$ $\bigcap_{n} G_{n}=e$ and $\sigma_{G / G_{n}}$ is a Bernoulli group endomorphism. So $h\left(T_{\zeta\left(G_{n}\right)}\right)=h(S)+h\left(\sigma_{G / G_{n}}\right)$ for all $n$ and taking limits (\$1.4) gives $h(T)=h(S)+h(\sigma)$.

4. Completion of proof. The proof is completed now in the manner of Rohlin [6] and Juzvinskir [2]; it must be shown that an arbitrary compact group $G$ breaks down into sequences of factor groups on which endomorphisms of the types dealt with in $\$ \$ 2$ and 3 are induced. The "proof by steps" procedure of $\$ 1.3$ can then be applied. Throughout this section, $C$ will denote the connected component of the identity of $G$ and $Z$ will denote the centre of $C$; both $C$ and $Z$ are completely invariant under any endomprphism $\sigma$ of $G$.

4.1. Totally disconnected groups. The first step is easy: $G / C$ is totally disconnected (addition theorem for $\sigma_{G / C}$ given by $\$ \$ 2.3$ and 2.4).

4.2. Connected groups with trivial centres. The next step will be $\sigma_{C / Z}$; this will require two results of Juzvinskir [2] which are reproduced in [4, Appendix B]. The first is that $C / Z$ has a trivial centre and the second is the following:

LeMma. An endomorphism $\rho$ of a compact separable connected group $H$ whose centre is trivial is the direct product of a Bernoulli group automorphism $\rho_{1}$, a Bernoulli 
group endomorphism $\rho_{2}$ and $\rho_{3}$ which is a direct product of automorphisms of semisimple Lie groups.

The addition theorem has been proved for Bernoulli group automorphisms and endomorphisms (\$3.2) and for endomorphisms of Lie groups ( $\$ 2.3$ and 2.5). So the (infinite if necessary) proof by steps procedure gives the addition theorem for $\rho$ as in the lemma. So the $\sigma_{C / Z}$ step is permissible. This leaves $Z$.

4.3. Abelian groups. The endomorphism induced on $Z$ factored by its connected component of the identity can be dealt with as in $\$ 4.1$ and so it remains to prove the addition theorem for an endomorphism $\sigma$ of a connected abelian group $G$. The character group of $G$ will be denoted by $\Gamma$.

First of all it is assumed that $\Gamma$ is finitely generated with respect to $\sigma$, i.e. every element of $\Gamma$ is of the form

$$
\gamma_{1} p_{1}(\sigma)+\gamma_{2} p_{2}(\sigma)+\cdots+\gamma_{n} p_{n}(\sigma)
$$

where $\gamma_{1}, \gamma_{2}, \ldots, \gamma_{n}$ are fixed elements of $\Gamma$ and $p_{1}, p_{2}, \ldots, p_{n}$ are polynomials with integer coefficients. (Note that the adjoint of $\sigma$ is denoted by the same symbol but written on the right.)

There are two possible cases:

(i) For every $\gamma_{i}$, there exists a polynomial $q_{i}$ such that $\gamma_{i} q_{i}(\sigma)=0$. In this case, $\Gamma$ is of finite rank. So $G$ is finite dimensional and the addition theorem for $\sigma$ is given by $\$ 2.6$.

(ii) For some $\gamma_{i}, \gamma_{i} q(\sigma) \neq 0$ for all possible polynomials $q$. Let $\Gamma_{i}$ be the subgroup generated by $\gamma_{i}$, let $\Omega$ be the smallest subgroup containing $\Gamma_{i}$ which is invariant under $\sigma$ and let $H$ be the annihilator of $\Omega$. The condition given implies that $\Gamma_{i} \sigma^{a} \cap \Gamma_{i} \sigma^{b}=e$ for $a \neq b$ and so $\Omega$ is the direct sum (finite numbers of nonzero terms) of $\Gamma_{i}, \Gamma_{i} \sigma, \Gamma_{i} \sigma^{2}, \ldots$, each of which is isomorphic to the integers. Its dual $G / H$ is the direct sum (unrestricted) of a one-way infinite sequence of circles and $\sigma_{G / H}$ is a Bernoulli group endomorphism with the circle as a group of states.

$h\left(\sigma_{G / H}\right)=\infty[3, \S 9.10]$, and so the addition theorem for $\sigma_{G / H}(\S 3.2)$ gives

$$
h\left(T_{\zeta(H)}\right)=h(S)+h\left(\sigma_{G / H}\right)=\infty .
$$

By 1.1, (i), $h(T) \geqq h\left(T_{\zeta(H)}\right)=\infty, h(\sigma) \geqq h\left(\sigma_{G / H}\right)=\infty$. Hence, $h(T)=h(S)+h(\sigma)$.

This proof is a much modified version of $[2, \S 8.4]$.

The restriction on $\Gamma$ is removed now.

Lemma (Rohlin [6, \$4.3], REPROduCED IN [4, APPENDix C.3]). If $\sigma$ is an endomorphism of a compact separable abelian group $G$, then $G$ contains a sequence $G=G_{0} \supset G_{1} \supset G_{2} \supset \cdots$ of $\sigma$-invariant closed subgroups such that $\bigcap_{n} G_{n}=e$ and the dual group of $G / G_{n}$ is finitely generated with respect to $\sigma_{G / G_{n}}$ for all $n$.

This lemma and the preceding work give $h\left(T_{\zeta\left(G_{n}\right)}\right)=h(S)+h\left(\sigma_{G / G_{n}}\right)$ for all $n$ and on taking limits (\$1.4), this becomes $h(T)=h(S)+h(\sigma)$.

This completes the proof of the addition theorem. 


\section{Applications.}

5.1. Group endomorphisms. If $\sigma$ is an endomorphism of a compact group $G$ onto itself and $H$ is a completely invariant closed subgroup, then $\sigma$ can be written as a suitable skew-product (see $\S 1.2$ ) and so $h(\sigma)=h\left(\sigma_{G / H}\right)+h\left(\sigma_{H}\right)$. For $H$ normal, this is the result of Juzvinskir [2], mentioned in the introduction. The addition theorem can be applied in a similar fashion to affine transformations.

5.2. Nilmanifolds. The addition theorem is used by W. Parry [7] to calculate the entropy of an automorphism of a nilmanifold. Suppose that $T^{\prime}$ is a group automorphism of a connected and simply connected nilpotent Lie group $N$ (lower central series $N=N_{0} \supset N_{1} \supset N_{2} \supset \ldots \supset N_{k-1} \supset N_{k}=e$ ) which takes a uniform discrete subgroup $D$ onto itself; the (left) coset space $N / D$ is a compact manifold known as a nilmanifold. $T^{\prime}$ induces a measure-preserving transformation on $N / D$ known as an "automorphism".

Let $T_{r}$ be the transformation induced on $N / N_{r} D$ and let $\sigma_{r}$ be the transformation induced on $N_{r-1} D / N_{r} D . T_{r}$ can be regarded as a skew-product of $T_{r-1}$ and $\sigma_{r}$ for $r=1,2, \ldots, k$, and so applying the addition theorem for each $r$, it follows that

$$
h(T)=h\left(\sigma_{1}\right)+h\left(\sigma_{2}\right)+\cdots+h\left(\sigma_{r}\right) .
$$

$\sigma_{r}$ is an automorphism of a torus and so

$$
h\left(\sigma_{r}\right)=\sum_{\left|\lambda_{i}\right|>1} \log \left|\lambda_{i}\right|
$$

(see [4] for references), the $\lambda_{i}$ 's being the eigenvalues of $\sigma_{r}$. Hence the entropy of $T$ can be expressed in the form

$$
h(T)=\sum_{\left|\mu_{i}\right|>1} \log \left|\mu_{i}\right|
$$

where the $\mu_{i}$ 's are the eigenvalues of the differential of $T$ at the identity coset of $N / D$.

\section{REFERENCES}

1. L. M. Abramov and V. A. Rohlin, The entropy of skew product measure-preserving transformations, Vestnik Leningrad. Univ. 17 (1962), 5-13; English transl., Amer. Math. Soc. Transl. (2) 48 (1965), 255-265. MR 25 \#4076.

2. S. A. Juzvinskiĭ, Metric properties of endomorphisms of compact groups, Izv. Akad. Nauk SSSR Ser. Mat. 29 (1965), 1295-1328; English transl., Amer. Math. Soc. Transl. (2) 66 (1968), 63-98. MR 33 \#2798.

3. V. A. Rohlin, Lectures on the entropy theory of measure preserving transformations with invariant measure, Uspehi Mat. Nauk 22 (1967), no. 5 (137), 3-56= Russian Math. Surveys 22 (1967), no. 5, 1-52. MR 36 \#349.

4. R. K. Thomas, Metric properties of transformations of G-spaces, Trans. Amer. Math. Soc. 60 (1971), 103-117.

5. S. Helgason, Differential geometry and symmetric spaces, Pure and Appl. Math., vol. 12, Academic Press, New York, 1962. MR 26 \#2986. 
6. V. A. Rohlin, Metric properties of endomorphisms of compact commutative groups, Izv. Akad. Nauk SSSR Ser. Mat. 28 (1964), 867-874; English transl., Amer. Math. Soc. Transl. (2) 64 (1967), 244-252. MR 29 \#5955.

7. W. Parry, Ergodic properties of affine transformations and flows on nilmanifolds, Amer. J. Math. 91 (1969), 757-771.

Birkbeck College, University of London,

LONDON, ENGLAND 